\title{
Politique
}

\section{Les femmes en politique, encore une exception}

\section{Carolle Simard et Denis Monière}

Numéro 5, hiver 1984

Femmes et pouvoir

URI : https://id.erudit.org/iderudit/040443ar

DOI : https://doi.org/10.7202/040443ar

Aller au sommaire du numéro

Éditeur(s)

Société québécoise de science politique

ISSN

0711-608X (imprimé)

1918-6584 (numérique)

Découvrir la revue

Citer ce document

Simard, C. \& Monière, D. (1984). Les femmes en politique, encore une exception. Politique, (5), 7-25. https://doi.org/10.7202/040443ar d'utilisation que vous pouvez consulter en ligne.

https://apropos.erudit.org/fr/usagers/politique-dutilisation/ 


\title{
Les femmes en politique, encore une exception
}

\author{
Entrevue avec Nicole Boily \\ Réalisée par Carolle Simard et Denis Monière
}

Nicole Boily quittait en aồt 83 le poste de directrice du cabinet de la ministre déléguée à la condition féminine, madame Pauline Marois. Militante de longue date du Parti québécois, elle participa à la campagne $d u$ référendum comme présidente du comité du oui d'Outremont et fut à deux reprises candidate dans ce comté à l'automne 80 et au printemps 81. Active au sein des comités d'école, elle siégea à l'exécutif de la Commission scolaire Ste-Croix de 77 à 80 et assuma la responsabilité de directrice générale de la Fédération des Femmes du Québec de 77 à 81. Ces diverses activités avaient été précédées d'une implication de près de quinze ans comme professeure et chercheure en bistoire de l'art et en éducation des adultes.

R.P.: Nous avons pensé intituler cette entrevue, «Les femmes en politique, encore une exception", que pensez-vous de cette affirmation?

N.B.: Je dirais que c'est de l'ordre de l'évidence. Il y a au Québec actuellement deux femmes ministres, $6 \%$ de députées, une directrice de cabinet sur 26. Quant à la haute fonction publique, c'est dans la même infime proportion que l'on retrouve les femmes. Les femmes sont donc encore l'exception dans les postes décisionnels à l'inverse de leur présence dans le militantisme politique. 
$R . P$.: Donc, la participation des femmes est plus intense au niveau des partis, au niveau du militantisme, et il y aurait un barrage lorsqu'on passe à des niveaux décisionnels?

N.B.: Les femmes sont traditionnellement absentes ou exclues des centres décisionnels des partis politiques même si ces dernières années un effort est fait pour s'assurer au moins d'une représentation minimale, à la fois parce que la situation devenait inconvenante et aussi, et surtout, parce que les femmes elles-mêmes font en sorte de prendre leur place. Par ailleurs, les femmes sont les piliers du militantisme politique quand il s'agit du financement, du recrutement, du travail d'élection, c'est-à-dire dans toutes les opérations qui réclament temps, patience et abnégation, travail dans l'ombre. Le même phénomène existe dans les organismes bénévoles et les groupes populaires. En effet, une étude récente a démontré que les postes-clefs des conseils d'administration des mouvements bénévoles mixtes comme la Croix-Rouge ou la Société canadienne du cancer ou même des groupes populaires sont bien davantage l'apanage des hommes que des femmes.

R.P.: Quels sont les facteurs de ce blocage?

N.B.: À mon avis, ce blocage s'explique fondamentalement par le phénomène de discrimination systémique que l'on peut observer dans tous les secteurs d'activité de notre société. Cette discrimination résulte de la division traditionnelle des rôles qui confine les femmes dans la vie privée et projette les hommes vers la vie publique. Ainsi, pour les postes de direction, on a «un» modèle, soit celui d'une personne publiquement connue, ayant un certain prestige, correspondant aux normes traditionnelles et s'y conformant. Alors, où est la place des femmes dans cette vision stéréotypée sinon dans les humbles tâches d'exécution?

R.P.: Donc, en politique comme ailleurs, les fonctions d'exécution continuent d'être assumées par des femmes, tandis que les fonctions de direction, qui se déplacent par exemple du parlement à l'exécutif ou à la haute fonction publique, demeurent fermées aux femmes. Que pensez-vous d'une politique importante mise de l'avant par 
le gouvernement du Parti québécois afin de favoriser la promotion des femmes à travers la fonction publique?

N.B.: Bien sûr, des mesures ont été mises en place à la fonction publique québécoise, des programmes d'égalité en emploi ont été créés avant même la nouvelle législation sur les programmes d'accès à l'égalité prévus maintenant dans la Charte québécoise des droits et libertés de la personne. Cependant, la résistance a été très forte car les hommes ont craint la perte des privilèges qu'ils considéraient comme des droits acquis de toute éternité et pour toujours. On peut citer, à titre d'exemple, la réaction de fonctionnaires du ministère de l'Environnement qui se sont opposés à certains de ces programmes gouvernementaux favorisant la promotion des femmes en intentant des poursuites judiciaires, s'estimant lésés dans leurs droits. Tout le monde est d'accord quand il s'agit d'affirmer le principe d'égalité ou la nécessité de rattrapage pour les femmes, mais lorsqu'on entreprend la concrétisation de ces principes, il en va tout autrement. À ce phénomène de résistance s'ajoute la conjoncture économique qui a entraîné des restrictions budgétaires limitant le nombre d'ouvertures de postes. Enfin, il faut aussi souligner que les femmes qui accèdent à des postes de commande doivent se qualifier de façon tout à fait exceptionnelle et encore selon le modèle masculin. C'est ce qu'on peut appeler une discrimination invisible mais profondément insidieuse.

\section{LA FEMME EST PORTEUSE D'UN NOUVEAU MODÈLE}

R.P.: Mais une des stratégies du gouvernement semble être d'augmenter la représentation chiffrée des femmes à différents niveaux. Que pensez-vous de cette stratégie? Est-ce une façon d'intégrer les femmes dans la politique?

$N . B$.: La représentation chiffrée des femmes ou le pourcentage d'augmentation est souvent trompeur. Qu'est-ce qu'une augmentation de $50 \%$ ou même $100 \%$ quand il s'agit de 2,3 ou 5 individus. C'est quand on analyse l'ensemble de la pyramide que l'on a une idée plus juste de la situation. On voit alors que les 
changements sont très lents. D'ailleurs, des études récentes, comme la vôtre, Carolle Simard, l'ont bien démontré.

On ne peut pas nier que la stratégie gouvernementale soit d'augmenter le nombre de femmes et le mouvement est amorcé mais grâce, particulièrement, à des femmes de mieux en mieux formées, de plus en plus conscientes, sensibilisées et décidées à prendre leur place. Il est donc évident que le gouvernement ne peut aller à contre courant et s'aliéner une partie de la population sans paraître réactionnaire. Il est de bon ton actuellement de nommer des femmes à des postes relativement importants. On est cependant très prudent dans ces nominations, on craint les «féministes» qui dérangent par leurs remises en question et leur volonté d'introduire de nouveaux modèles.

R.P.: Vous croyez donc que la femme porte avec elle un nouveau modèle et que sa participation au processus décisionnel changerait le fonctionnement du politique. Le fait d'être une femme seraitil un antidote aux relations de pouvoir et d'autorité?

$N . B .:$ Je crois, en effet, que la femme est porteuse d'un nouveau modèle si elle écoute sa différence, si elle fonctionne selon sa propre échelle de valeur sans être teintée par le modèle masculin. La pratique du pouvoir pourrait ainsi être différente s'il y avait un nombre significatif de femmes, l'on pourrait voir apparaître des changements au fonctionnement du politique. Déjà à l'Assemblée nationale, les relations qui s'exercent entre les quelques femmes des deux côtés de la Chambre marquent cette différence. Les modifications pouvant intervenir ne provoqueront pas une absence de pouvoir et d'autorité mais les rapports de force pourront se manifester de façon différente en vertu de nouvelles valeurs. Il est cependant évident qu'un changement aussi fondamental de mentalité marquée par une tradition millénaire ne pourra s'effectuer en quelques années, c'est tout le rapport dominants-dominés qui est ici en cause.

R.P.: Est-ce que vous diriez que parce que les femmes sont socialement déterminées à occuper certaines fonctions qu elles sont 
aussi socialement déterminées à aborder le champ politique ou le rapport au politique, ce qui fait qu'elles entrent en politique de manière différente, non pas parce qu'elles sont biologiquement déterminées, mais parce qu'elles sont socialement déterminées, parce que leur expérience notamment leur rapport aux enfants, leur fait découvrir tout un aspect de la vie, des rapports entre les êtres, dont généralement les hommes ne sont pas conscients?

$N . B$. : Je crois sincèrement que les femmes sont plus que socialement déterminées mais que c'est aussi psychologiquement et dans tout leur être qu'elles sont différentes et qu'elles abordent les problèmes selon leur perception.

$R . P .:$ Est-ce que vous avez des exemples pour illustrer ce fait?

N.B.: Mon expérience personnelle m'a permis d'arriver à cette constatation. J'ai travaillé tout autant dans des milieux essentiellement masculins que dans des milieux féminins. J'ai remarqué une différence de fonctionnement. Les femmes sont beaucoup plus concrètes. Leur analyse d'une situation part d'une expérience vécue et leur champ de connaissance et d'intervention s'élargit conséquemment dans un mouvement circulaire. Les hommes ont une vision plus synthétique. Alors que la conceptualisation vient à la suite d'expériences vécues chez les femmes, elle est prioritaire chez les hommes et de celle-ci découleront des applications concrètes. R.P.: Les hommes ont tendance à exprimer une pensée plus globale alors que les femmes auraient une tendance à exprimer une pensée plus empirique?

N.B.: Je serais assez d'accord avec cette formulation. La quotidienneté des femmes, les tâches qu'elles ont accomplies depuis des millénaires, qu'elles soient ou non sur le marché du travail, leur ont aussi permis de développer cette attitude.

$R . P .:$ Donc, si on résume, le blocage que l'on a constaté peut en partie résulter de cette différence de vision du monde ou de conception de fonctionnement du pouvoir, cela pourrait être une des explications. La difficulté des femmes de s'intégrer dans la fonction publique et dans les postes de pouvoir serait due au fait que les hommes imposent leurs normes ou leurs règles du jeu. 
N.B.: Bien sûr, les hommes fixent et imposent leurs normes et les règles du jeu, ils laissent peu de place à de nouveaux modèles. Mais j'ajouterais que consciemment ou inconsciemment, les hommes sont peu enclins à partager le pouvoir qu'ils détiennent. L'égalité est une notion abstraite mais le partage est une réalité concrète plus difficile à vivre.

\section{LES INNOVATIONS DES ANNÉES '70}

R.P.: Mais cependant, depuis quelques années on constate qu'il y a une ouverture de ce point de vue, au niveau des appareils d'État. Il ya eu un grand progrès si on compare aux années 50 et 60 , est-ce que vous pourriez présenter les innovations institutionnelles qui ont marqué l'entrée des femmes dans l'appareil d'État?

$N . B$. : Ce sont les années 70 qui ont marqué le début d'une période de progrès pour les femmes. Les années 60 avaient vu l'élection des deux premières femmes à l'Assemblée nationale, d'abord Claire Kirkland-Casgrain en 1961 et quelques années plus tard Lise Bacon. Il faut attendre 1976 pour que le nombre d'élues passe à 5 et l'élection de 1981 où 8 femmes sont élues. Cette progression à pas de tortue est similaire dans la haute fonction publique. La force d'influence et de pression de ces femmes, même si elles le voulaient, ne pouvait et ne peut être très puissante. Même si Claire Kirkland-Casgrain a marqué des points pour les femmes par des modifications au code civil, on ne peut pas dire que les années 60 aient été fertiles en innovations institutionnelles pour les femmes. Les véritables changements s'amorcent dans les années 70 , moment qui correspond à une meilleure organisation des femmes entre elles. Les gains sont le résultat des luttes entreprises par ces groupes de pression.

$R . P .:$ Le progrès ne vient pas des partis politiques?

$N . B$.: Le progrès vient de la pression des femmes organisées à l'extérieur mais aussi à l'intérieur des partis politiques. Aucun changement n'est venu spontanément des hommes, détenteurs 
des pouvoirs, qui auraient pris conscience de la situation anormale et injuste qui était faite aux femmes. Au Québec, la première lutte politique organisée fut celle pour l'obtention du droit de vote, obtenu finalement en 1940 . Les revendications politiques articulées ont redémarré à la fin des années 60 quand les femmes ont commencé à se regrouper pour faire pression. Ces luttes ont permis entre autres la création, en 1973, du Conseil du Statut de la femme, organisme consultatif du gouvernement voué à l'amélioration de la condition de vie des femmes qui avait été réclamé par la Fédération des Femmes du Québec. À la même époque, à Ottawa, le gouvernement central avait mis sur pied une Commission d'enquête sur la situation de la femme au Canada, la Commission Bird, qui a entraîné la naissance du Conseil consultatif canadien sur la situation de la femme. C'était les premières fois que l'on mettait le dossier «femmes» sur la carte. Auparavant, on parlait «famille» pour parler «femmes».

Nous avons vécu ensuite l'année internationale des femmes en 1975 avec son "petit» cortège de coups de chapeau à la cause des femmes.

À partir de 1976, un certain nombre de législations fondamentales ont été adoptées: congés de maternité, loi sur les normes minimales de travail, réforme du droit de la famille, programmes d'accès à l'égalité qui comptent parmi les plus importantes. Le Parti québécois avait un comité des conditions féminines très actif qui avait fait inclure dans le programme du parti des mesures progressistes pour les femmes. Ce qui n'a pas été sans effet sur le programme gouvernemental de ce début de mandat. C'est aussi à ce moment qu'on a commencé à songer, au sein de l'appareil d'État, à des programmes d'égalité en emploi pour les femmes dans la fonction publique.

R.P.: Diriez-vous que ce gouvernement a favorisé les femmes en tant qu'acteur politique, que des mesures ou des politiques ont favorisé les femmes? On peut penser par exemple à ce qui se passe actuellement, aux audiences publiques sur la réforme du mode 
de scrutin où l'État ne s'est pas privé d'utiliser l'argument féministe pour favoriser cette réforme?

$N . B$. : Les gouvernements ont presque inévitablement une tendance électoraliste et récupératrice, je vous le concède. Ainsi, le gouvernement arrivé au pouvoir en 1976 pouvait encore se permettre du développement; de plus on était encore sous l'influence de l'année internationale des femmes. Si on ajoute à ces conditions, la présence d'une ministre comme Lise Payette qui était une fonçeuse, on trouve des circonstances qui, pendant quelques années, je dirais jusqu'au référendum, ont amené certains changements même s'ils n'étaient pas à la mesure des demandes des femmes ni avec la rapidité jugée nécessaire. Il faut cependant admettre qu'il y a eu un certain cheminement, un certain progrès. Après le référendum et avec la nouvelle conjoncture économique, les choses ont changé.

R.P.: Vous considérez que la création d'un ministère de la condition féminine a été le principal acquis pour les femmes?

$N . B$.: Pas nécessairement le principal, mais un acquis d'autant plus qu'il avait le statut de ministère d'État avec ceux du développement économique, développement social, développement culturel et de l'aménagement. C'était et c'est toujours un instrument utile pour la cause des femmes. Actuellement, je crois sincèrement que s'il n'y avait pas de ministre à la Condition féminine, on vivrait des reculs encore plus importants que ceux qui sont déjà intervenus.

$R . P$. : N'est-ce pas une façon justement de favoriser la participation de la femme comme exception en créant un ministère ghetto? N'est-ce pas une mesure dilatoire qui évite au gouvernement de prendre ses responsabilités dans l'ensemble des ministères?

N.B. : À mon avis, non, car la ministre à la Condition féminine n'a pas un ministère sectoriel, elle n'a pas de pouvoir direct sur les mesures prises dans des champs sectoriels.

R.P.: Elle n'a pas de pouvoir?

N.B.: Entendons-nous, elle n'a pas de prise première et directe sur les législations ou les programmes élaborés par les divers 
ministères. Son pouvoir en est un d'influence sur ses collègues qu'elle tente de responsabiliser. Si la ministre à la Condition féminine avait la responsabilité unique de tout ce qui concerne les femmes, c'est là qu'elle serait ghettoisée et marginalisée. Je m'explique. Dans ces conditions, aucun autre ministère ne se soucierait de la situation particulière des femmes, on ne serait que trop heureux de lui en laisser l'entière responsabilité. De plus, comment arriver à une continuité et une cohérence dans des politiques qui sont morcelées. Cela serait au détriment des femmes. La nécessité d'une ministre à la Condition féminine se défend par l'horizontalité de son dossier. Elle doit voir à ce que la particularité et les besoins spécifiques des femmes soient pris en charge par tous les ministères. Elle peut aussi initier certaines politiques ou certains changements. C'est son rôle de faire prendre conscience à ses collègues que ce ne sont pas uniquement des mesures isolées, correspondant à la correction d'injustices flagrantes, qui doivent être apportées mais que doit intervenir un changement fondamental d'approche pour un statut d'égalité dans la différence. Ce changement d'attitude, de mentalité est peut-être ce qu'il y a de plus difficile à faire passer.

\section{MINISTRE DE LA CONDITION FÉMININE: RÔLE ACROBATIQUE}

R.P.: Dans ce contexte là, est-ce que la fonction de la ministre à la Condition féminine, n'est pas une mission impossible? Puisque en fait ce sont des cas extrêmes qui semblent frapper les politiciens, alors que le féminisme a démontré que ce n'était pas des cas, que c'était la situation fondamentale des femmes qui était à revoir, qui était à changer.

$N . B$.: Effectivement, c'est une situation très difficile que celle de la ministre à la Condition féminine. Ce n'est pas de tout repos car elle est le plus souvent l'opposition à l'intérieur du pouvoir. La capacité d'influence et de persuasion s'exerce aussi dans une relation de pouvoir, un rapport de force tout autant que dans un 
effort pour changer les mentalités. Par ailleurs, il faut aussi dire que le champ d'intervention est plus large que celui du seul Conseil des ministres. La ministre a donc autour d'elle pour l'assister, outre son cabinet politique, un secrétariat composé d'une secrétaire générale associée, ayant rang de sous-ministre, et des fonctionnaires qui elles aussi ont un rôle à jouer pour la promotion de politiques favorables aux femmes et pour la création d'un réseau de solidarité. Réseau formel qui existe par la présence de répondantes à la condition féminine dans les principaux ministères, mais aussi réseau plus informel. Il y a de nombreux exemples qui manifestent que la ministre et son entourage ont eu un rôle indispensable dans la réorientation de mesures à l'intérieur de législations ou de programmes. Pour parler de cas précis, il est certain que la réforme du droit de la famille ou que les modifications à la Charte québécoise des droits et libertés de la personne n'auraient pas pris la même tournure sans l'intervention de la ministre et de son entourage appuyée par le C.S.F. et les groupes de femmes. R.P.: Quels sont les rapports des groupes de femmes avec le ministère de la Condition féminine? Autrement dit, lorsque ce ministère définit les priorités et les orientations, qui sont les intervenants qui orientent ces priorités et ces interventions, estce que ce sont les groupes de femmes, est-ce que ce sont les groupes de femmes à l'intérieur du parti ou est-ce que ce sont les fonctionnaires, le personnel de cabinet qui conçoivent ces priorités, ou la ministre elle-même?

N.B. : Le rapport du ministère avec les groupes de femmes doit, à mon avis, être très étroit. C'est un des moyens pour garder des liens avec la réalité du terrain. De plus, la pression des groupes de femmes est un soutien de l'extérieur à une action à l'intérieur, même si parfois c'est lourd et dérangeant. Les femmes ne sont pas toujours conscientes de la force qu'elles peuvent avoir lorsqu'elles se concertent sur des points précis. En ce qui a trait à l'établissement des orientations et des priorités, elles sont définies par la ministre avec l'aide et le support de ses conseillères, tant les fonctionnaires du secrétariat que le personnel politique de son cabinet. Il ne 
faudrait pas non plus négliger le rôle conseil que joue le Conseil du Statut de la femme. Par ailleurs, il est évident que les contacts avec les groupes de femmes, la connaissance de leurs besoins et leurs revendications de même que l'information sur les grands projets gouvernementaux et la conjoncture politique sont aussi des guides dans l'élaboration des priorités ministérielles en matière de condition féminine. Je voudrais spécifier ici que je parle de ce que j'ai connu et qu'il pourrait en être tout à fait autrement selon la ministre et son entourage.

R.P.: Toutes des femmes?

$N . B$. : Toutes des femmes, avec épisodiquement la participation d'un représentant de la gent masculine au cabinet de la ministre. Ce qui était de première importance, au moment où j'étais là, c'était ce contact avec les femmes vivant différentes situations, la liaison entre le pouvoir politique et les groupes de base.

$R$.P. : En tant que membre de cabinet politique, participiez-vous directement à ces groupes de pression ou étiez-vous identifiée clairement comme personnel de cabinet?

$N . B$. : Je crois qu'il est essentiel d'identifier clairement le rôle et la place de chacun, personnel politique et groupes de pression. Les unes et les autres ont des fonctions différentes et distinctes, ce qui n'empêche nullement les contacts et les liens. Il faut éviter toute situation d'ambiguité qui pourrait donner lieu à des manipulations ou à de la récupération dans un sens comme dans l'autre. Ayant vécu l'une et l'autre situation, j'estime possible et nécessaire l'identification des rôles respectifs.

\section{LE PROBLÈME DE LA DOUBLE ALLÉGEANCE}

R.P. : Comment avez-vous réglé ce problème de la double allégeance, vous Nicole Boily, militante, féministe, au Parti Québécois, et ensuite comme membre d'un appareil politique?

$N . B$.: Le problème de la double allégeance est très sérieux et difficile à concilier. Je dirais que personnellement je me suis laissée guider par mon instinct et aussi par mon sens de l'éthique. Je 
dois dire que j’ai accepté un poste politique dans le cadre du dossier particulier de la condition féminine après 4 ans à la coordination de la Fédération des Femmes du Québec. J'avais travaillé à l'amélioration de la condition de vie des femmes à l'extérieur du gouvernement, je souhaitais, puisqu'on m'en offrait l'occasion, faire un bout de chemin à l'intérieur. L'allégeance féministe était donc prioritaire. Ceci dit, tout n'est pas réglé pour autant, car éthiquement j'avais à me situer dans le cadre du parti auquel j'appartenais, du gouvernement dans lequel j'avais à fonctionner et aussi de l'appareil d'État en place. Cette situation de la double allégeance n'est-elle pas vécue à des degrés divers, selon des modalités différentes par beaucoup de féministes dans des secteurs autres que politiques? Je reconnais cependant qu'elle est particulièrement difficile à assumer en politique et qu'elle entraîne beaucoup de frustrations.

R.P.: Peut-on vous demander pour quelles raisons vous avez démissionné?

N.B.: Ce fut une décision douloureuse et difficile que j'ai prise après un long moment de réflexion. J'avais l'impression d'interrompre un processus alors qu'il y avait encore des actions à mener à terme. Cependant, il m'est apparu de plus en plus clairement, et surtout à cause du poste que j'occupais, que je ne pouvais plus défendre un gouvernement, des politiques avec lesquelles je n'étais plus en accord et qui allaient à l'encontre de ce que je pensais et souhaitais pour le Québec. Ces désaccords s'accumulaient et touchaient des aspects fondamentaux de la gestion de l'État comme la politique des négociations, les choix dans le domaine de l'emploi, dans le domaine de l'éducation des adultes sans compter l'absence de politiques cohérentes en ce qui concerne les femmes, tout ceci pour ne citer que quelques exemples. De plus, les blocages étaient de plus en plus fréquents et forts pour la mise en place de mesures favorables aux femmes aussi bien dans les politiques que dans les gestes du quotidien. On était régi par des politiques à la pièce, de façon ponctuelle et sans perspective. J'ai donc décidé de partir. 
R.P.: Dans cette ligne de pensée, comment analysez-vous le pouvoir d'influence d'une ministre femme dans un cabinet qui doit traiter de dossiers qui ne relèvent pas de la condition féminine? Il y a des questions sur lesquelles le problème homme/femme n'intervient pas, est-ce qu'une femme ministre a autant d'influence dans ces dossiers neutres disons que si c'était un homme, est-elle sur le même pied d'égalité à ce niveau-là au cabinet?

N.B. : Comme préalable, je dirais que les femmes étant très minoritaires au Conseil des ministres, elles doivent avec difficulté faire leur place, faire la preuve qu'elles sont «bonnes», capables de remplir la tâche qu'on leur a confiée. N'est-ce pas d'ailleurs le témoignage apporté par presque toutes les femmes ministres et ex-ministres et même par les femmes députées. Elles doivent faire leurs preuves pour atteindre ce degré d'égalité. N'est-ce pas significatif que l'on spécifie d'une femme ayant un poste de responsabilité qu'elle est intelligente, qu'elle travaille bien, alors qu'on ne juge pas nécessaire de le faire pour un homme pour qui ces qualités sont jugées normales.

Pour revenir à votre question, je dirais qu'au-delà des remarques que j'ai faites précédemment, la situation est variable selon les individus et selon les dossiers. L'important est que la ministre réussisse à s'imposer dans son propre secteur d'intervention. Pour prendre un cas particulier, citons celui de Mme Payette qui est entrée au Cabinet avec le dossier des institutions financières et coopératives où elle a réussi à faire passer la loi sur l'assuranceautomobile. Elle a dû avoir un pouvoir d'influence auprès de ses collègues qui n'étaient pas nécessairement tous favorables aux mesures qu'elle préconisait.

Quant à la ministre à la Condition féminine, lorsqu'elle s'intéresse à d'autres dossiers que le sien, elle est relativement sur le même pied que ses collègues soit dans le camp des juniors ou des seniors. $\mathrm{Sa}$ formation et ses expériences professionnelles antérieures peuvent aussi ajouter à sa crédibilité puisque cela correspond au modèle masculin de promotion ascendante.

R.P.: Elle est tout de même membre du comité des priorités? 
N.B.: Oui, la ministre à la Condition féminine est membre du comité des priorités. Ouf? oui encore, mais après quelles tribulations! C'est en effet un lieu de pouvoir puisqu'on y définit des politiques et des orientations majeures pour le gouvernement mais c'est aussi un lieu où l'on peut puiser beaucoup d'informations. En ce qui a trait au statut que la ministre peut y avoir, là aussi, il faut qu'elle y prenne sa place.

\section{UNE DÉMARCHE DE CARRIÈRE COHÉRENTE: ADHÉSIONS ET RUPTURES}

$R . P .:$ Est-ce que vous, personnellement, vous avez été déçue par votre expérience? Vous avez dit précédemment, que bien sûr la création du ministère a été très positive, par contre vous avez démissionné parce que la politique globale du gouvernement n'allait pas dans la direction que vous souhaitiez et probablement qu'au ministère même vous sentiez des résistances, des obstacles?

$N . B$.: Je ne suis absolument pas déçue par l'expérience, car je ne m'étais fait aucune illusion, je dirais que j'ai même adoré ce genre de travail et que j'y ai beaucoup appris sur le fonctionnement quotidien de l'État, sur les modalités de prises de décision d'un gouvernement. J'ai par ailleurs essayé de faire le plus honnêtement possible mon travail pour faire avancer le dossier des femmes, cela dans la mesure des possibilités qui étaient à ma disposition. De plus, pour une conseillère politique, la situation est privilégiée pour tenter de faire accepter certaines idées qui nous tiennent à coeur et qui peuvent éventuellement se traduire en politiques, surtout si la ministre endosse ces objectifs. On a aussi un accès direct aux cabinets des différents ministères et aussi à la machine administrative. À certains égards, ce sont des postes stratégiques qui, tout en étant différents, peuvent être plus intéressants et recelant plus de pouvoir d'influence que les postes de députés surtout dans notre système parlementaire actuel. Donc, personnellement, je retire, malgré certains frustrations, plus de positif que de négatif de cette expérience. Par ailleurs, au plan collectif, 
étant peu nombreuses à posséder cette expertise, j'espère pouvoir la partager, démystifier cette mécanique et inciter d'autres femmes à entrer en politique.

R.P.: Mais pourquoi avoir démissionné, pourquoi ne pas avoir cherché à aller ailleurs au sein du gouvernement, et si je vous pose cette question, c'est que madame Payette disait toujours: «les femmes en politique n'ont pas de stratégie de carrière» comme dans les milieux professionnels, elles sont toujours prêtes à démissionner et à aller ailleurs, à changer. Votre exemple nous rappelle ce que disait madame Payette effectivement dans son livre, les femmes ne semblent pas avoir de stratégie de carrière en politique, est-ce que vous êtes d'accord avec ça?

$N . B .:$ Je dirais que les femmes n'ont pas, ou peu d'entre elles, de stratégie de carrière tant en politique que dans d'autres domaines; ce qui ne veut pas dire qu'il n'y ait pas de cohérence dans leur démarche. Selon le modèle traditionnel, on conçoit la carrière selon une ligne verticale ascendante, c'est-à-dire qu'en partant de la base on doit accéder à des postes permettant de se hisser vers le sommet de la pyramide que ce soit dans l'entreprise privée ou l'entreprise publique. C'est principalement de cette façon que l'on détermine la réussite d'une carrière. Les femmes, pour bon nombre d'entre elles, n'ont pas cette démarche, ce qui n'implique pas, par ailleurs, qu'elles refusent des postes de responsabilités.

En ce qui me concerne, j'ai démissionné parce qu'en tant que conseillère politique j'étais étroitement associée et j'avais à défendre les politiques du gouvernement au pouvoir. N'étant plus en accord avec certaines orientations, le choix qui s'offrait à moi était ou bien de me ranger et mettre en veilleuse mes divergences ou bien démissionner. J'ai fait mon choix pour ma propre cohérence et aussi par sens de l'éthique tout en étant consciente que, ne faisant pas partie de la fonction publique, je n'étais pas reclassée dans l'appareil administratif.

R.P.: Plusieurs personnes, je ne dis pas nécessairement des hommes, mais je dis d'autres hommes, d'autres femmes auraient tenté 
d'aller chercher un autre emploi avant de démissionner, je pense que c'est une stratégie possible et vous ne l'avez pas fait?

N.B.: Effectivement. Je n'ai pas voulu profiter de relations ou de contacts que je pouvais avoir au moment où j'étais encore directrice de cabinet. Je n'ai nullement la prétention de porter un jugement sur l'une ou l'autre stratégie, j'ai tout simplement suivi mon modèle de fonctionnement à moi. Mon cas est celui de nombreuses femmes qui ont une carrière en dents de scie, dans un enchaînement d'adhésions et de ruptures. Il y a une cohérence dans cette démarche mais elle n'est pas nécessairement calquée sur le modèle traditionnel.

\section{LE FÉMINISME EST EN BONNE SANTÉ AU QUÉBEC}

R.P. : Pouvez-vous comparer la situation de la femme au Québec par rapport à d'autres pays?

N.B.: Globalement, la situation des femmes au Québec est tout à fait comparable, meilleure ou moins bonne selon les domaines, à la situation des femmes dans les autres pays. Particulièrement au cours des 10 dernières années on a fait beaucoup de rattrapage au Québec. Plusieurs législations et politiques ont permis de faire des progrès substantiels. Ce n'est sûrement pas une raison pour nous reposer sur nos lauriers, d'autant plus que les mentalités n'ont pas nécessairement évolué au même rythme et que l'application de ces législations ou politiques en est de ce fait ralentie. On peut dire toutefois que le féminisme est en bonne santé au Québec. Les femmes, après un moment d'essoufflement, sont à nouveau prêtes à défendre leurs droits et leur place pour évoluer vers une société qui soit organisée de plus en plus à leur image.

R.P.: Diriez-vous que les femmes québécoises sont fortes parce que les hommes québécois sont plus faibles, certains analystes étrangers ont dit que l'homme québécois avait été castré. Que pensez-vous de cette thèse?

$N . B$.: Je ne crois pas que les femmes québécoises tirent leur force de la faiblesse de leurs congénères hommes. La conjoncture politique, 
sociale et culturelle a peut-être permis une accélération de la prise de conscience des femmes et a ainsi accentué leur besoin de s'organiser en groupes de pression. Le mouvement des femmes et la résurgence de leurs luttes n'est d'ailleurs pas le seul fait du Québec, cette situation existe partout dans le monde occidental et même plus largement. On s'aperçoit d'ailleurs, que, toute proportion gardée, les problèmes rencontrés par les femmes sont assez similaires où qu'on soit, et que la résistance aux changements s'exprime partout par des hommes qui se sentent menacés.

\section{TRAVAILLER DANS TOUS LES MILIEUX, INVESTIR LES CENTRES DE DÉCISION}

R.P.: En terminant une dernière question. Étant donné votre expérience, votre connaissance des blocages, des résistances à l'intérieur du système disons-le «traditionnel» entre guillemets, estce que vous estimez que la promotion de la femme pourrait mieux se faire par la création d'un parti exclusivement féminin ou si on peut toujours faire progresser la cause des femmes à l'intérieur des partis mixtes?

N.B. : À mon avis, il ne faut exclure aucune voie. Tout d'abord, il faut voir que les femmes ne sont pas un bloc homogène et monolithique. Elles présentent des variantes tant dans leur pratique du féminisme que dans leur conception de la société à bâtir. Étant donné cette situation, je crois que les femmes doivent travailler dans tous les milieux qui leur conviennent que ce soit dans des partis politiques mixtes, traditionnels ou progressistes, que ce soit dans un parti politique féministe. Ce ne sera qu'en imposant et en affirmant leur présence et leurs perceptions partout et dans tous les milieux que les femmes amélioreront leur condition de vie et travailleront à leur promotion.

R.P.: Cette stratégie ne risque-t-elle pas de diviser les forces?

$N . B$. : Non, je ne le crois pas et de plus cette stratégie se développe dans le respect des différentes tendances existantes chez les femmes. Cette présence des femmes dans plusieurs partis peut permettre 
des percées sur plusieurs fronts, ce qui n'est pas négligeable et peut aussi stimuler l'émulation entre les divers partis politiques. À mon avis, ce n'est pas un éparpillement ou une division des forces mais tout simplement une stratégie réaliste tenant compte des forces en présence. Par ailleurs, cela n'exclut pas des solidarités ponctuelles des femmes des différentes tendances comme on a pu le voir ces dernières années. La solidarité des femmes est un phénomène important dont elles ont fait l'apprentissage et qui leur permet à un moment donné, sur des points concrets et circonscrits, de lier leurs efforts. On pourrait même penser, lors d'une prochaine élection, à un regroupement de candidatures indépendantes de femmes pouvant rallier dans quelques circonscriptions des femmes de tous les partis afin d'assurer la présence accrue mais aussi la voix des femmes à l'Assemblée nationale.

R.P.: Vous pensez qu'il est impossible de stimuler le Parti québécois de l'intérieur? Pourquoi faut-il être à l'extérieur pour faire pression et faire avancer la cause des femmes à l'intérieur du Parti québécois? N.B.: Je crois que les femmes à l'intérieur du Parti québécois ont un rôle à jouer pour faire avancer la cause des femmes dans ce parti, mais en même temps une pression extérieure peut être un soutien, un support pour ces femmes.

R.P.: Mais vous Nicole Boily, vous pensez que c'est plus efficace à l'extérieur?

N.B.: Personnellement, je pense être allée au bout d'une démarche, d'avoir tenté ce que je pouvais. Je ne suis plus intéressée pour le moment à militer à l'intérieur de ce parti qui a modifié trop sensiblement ses orientations de départ. Le programme du parti quant à son projet de société et quant à l'amélioration de la condition de vie des femmes s'est atrophié. Je continuerai à militer politiquement très certainement mais pas dans les rangs du Parti québécois.

R.P.: À la suite de votre intervention, est-ce que vous diriez que ce sont les femmes qui rejettent la politique et non pas la politique qui rejette les femmes, qu'est-ce que vous en pensez? 
$N . B$. : Les femmes ne rejettent pas la politique, elles veulent au contraire y accéder à part entière et aussi elles veulent pouvoir y fonctionner selon un modèle qui leur est propre, elles veulent que l'on prenne conscience de la situation à changer pour les femmes et par voie de conséquence pour les hommes et la société tout entière. D'ailleurs leur combat est essentiellement politique. Les femmes ont pris conscience qu'elles doivent investir les centres de décision et donc les lieux de pouvoir politique. Elles ont d'ailleurs manifesté leur impatience à cet égard lors du forum sur les questions économiques du Conseil du Statut de la femme en octobre dernier alors que près de 1500 femmes de toutes les régions du Québec étaient rassemblées.

Montréal, novembre 1983 